\title{
Pre-Nucleation Self-Assembly and Chiral Discrimination Mechanisms during Solution Crystallization of Racemic Diprophylline
}

\author{
Clément Brandel, ${ }^{*[a]}$ Yohann Cartigny, ${ }^{[a]}$ Gérard Coquerel, ${ }^{[a]}$ Joop H. ter Horst,${ }^{[b]}$ and Samuel Petit ${ }^{[a]}$
}

\begin{abstract}
The crystallization behavior of (RS)-Diprophylline (DPL) in two different solvents is investigated in order to assess the incidence of solvated pre-associations on nucleation, crystal growth and chiral discrimination. In the solvated state, Raman spectroscopy shows that dimeric associations similar to those depicted in the crystalline solid solution (ssRII) predominate in isopropyl alcohol (IPA), which may account for the systematic spontaneous nucleation of this crystal form from this solvent. By contrast, spontaneous nucleation in dimethylformamide yields the stable racemic compound $\mathrm{RI}$, consistently with the distinct features of the Raman spectrum collected in this solvent. A crystal growth study of ssRII in IPA reveals that the crystal habitus is impacted by the solution enantiomeric excess which is explained by an increased competition between homo and heterochiral pre-associations. This is supported by a molecular modelling study on the enantiomeric selectivity of the DPL crystal lattices. The combination of assessment methods on solution chemistry, nucleation and chiral discrimination provides methodological tools from which the occurrence of solid solutions can be rationalized.
\end{abstract}

\section{Introduction}

Crystallization of organic compounds is often an efficient separation process and is widely applied at laboratory and industrial scales for purification purpose..$^{[1,2]}$ In the specific case of chiral compounds, the establishment of robust crystallization processes requires knowledge about the type of heterogeneous equilibria occurring between the enantiomers, which can be gained through the construction of the binary phase diagram. ${ }^{[3-5]}$ Since the end of the $19^{\text {th }}$ Century, ${ }^{[3,6]}$ it has been highlighted that three basic situations can be encountered in such diagrams: ${ }^{[7,8]}$ (i) the racemic compound, consisting of a 1:1 defined compound of the two enantiomers usually related by centrosymmetry in the crystal lattice; (ii) the conglomerate, made of a mixture of enantiopure crystals and (iii) the solid solution, depicting various degrees of chiral discrimination giving rise to mixed crystals (Figure 1). The complexity of such diagrams increases if

[a] Dr. C. Brandel, Dr. Y. Cartigny, Prof. G. Coquerel, Prof. S. Petit Laboratoire SMS-EA3233, Université de Rouen, Place E. Blondel, F-76821, Mont Saint Aignan Cedex, France;

E-mail: clement.brandel@univ-rouen.fr

[b] Prof. J.H. ter Hors

University of Strathclyde, EPSRC Centre for Innovative Manufacturing in Continuous Manufacturing and Crystallization (CMAC), Strathclyde Institute of Pharmacy and Biomedical Sciences (SIPBS), Technology and Innovation Centre, 99 George Street, Glasgow G1 1RD, U.K. polymorphism $^{[9-11]}$ occurs in the system or if metastable equilibria are considered. ${ }^{[12-14]}$

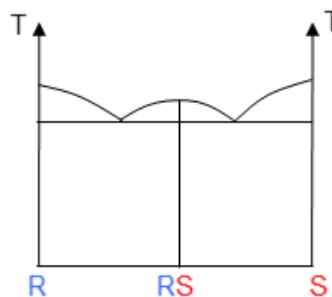

(a)
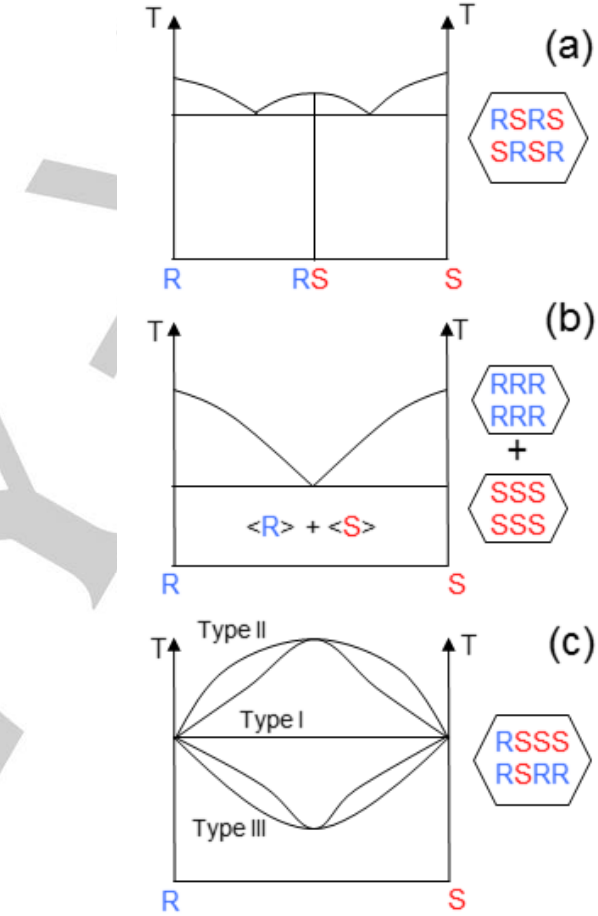

Figure 1. Schematic representation of the three archetypal phase diagrams encountered for enantiomeric mixtures: (a) racemic compound, (b) conglomerate and (c) the three types of solid solutions according to Roozeboom classification. ${ }^{[6]}$

Despite progress in the solid-state characterization of chiral systems within the last decades, ${ }^{[15-18]}$ the mechanisms of chiral selectivity in crystalline architectures are insufficiently understood. For instance, the prevalence of racemic compounds (depicted in ca. $90 \%$ of chiral systems) over conglomerates (less than $10 \%$ of occurrences) remains a challenging question. ${ }^{[19-21]}$ Concerning the infrequently identified enantiomeric solid solutions, the absence of chiral selectivity of the crystallographic sites provides valuable opportunities to investigate molecular mechanisms of chiral discrimination, in particular if this behaviour can be compared to that of a highly stereoselective racemic compound existing in the same system. About 35 years ago, Chion et al. ${ }^{[22]}$ underlined the role of two structural criteria regarding the formation of enantiomeric solid solutions: isosterism (i.e., the building units of opposite chirality are sterically equivalent) and isomorphism (i.e., substitution of one 
enantiomer by its counterpart in the crystal lattice preserves the main intermolecular interactions of the structure). Since then, few crystal structures of enantiomeric solid solutions have been reported and thoroughly described. ${ }^{[23-33]}$ In some cases, it can be observed that isosterism and isomorphism criteria are actually satisfied in relation to a conformational flexibility of the components, which provides sufficient degree of freedom in each enantiomer to mimic the conformation of its counterpart (i.e., conformational mimicry). Since molecular flexibility is also known to have a potential impact on crystallization behaviour and polymorphism (e.g. difficult nucleation, concomitant polymorphism, etc. ${ }^{[34-36]}$, scientists aim at rationalizing chiral discrimination during crystallization of an enantiomeric mixture by combining the analysis of stereoselectivity in the solid state, with insights into pre-nucleation species ${ }^{[37-40]}$ and their incidence on nucleation and crystal growth phenomena. ${ }^{[41,42]}$

As shown in Figure 2, our recent investigation of the binary system formed between Diprophylline (DPL) enantiomers revealed a complex situation containing no less than four crystal forms. ${ }^{[43]}$ Indeed, this chiral derivative of theophylline exhibits two polymorphic forms of pure enantiomers ( $E I$ is the stable form $\mathrm{mp}=165.7^{\circ} \mathrm{C}$ and ssEII, $\mathrm{mp}=124.0^{\circ} \mathrm{C}$ (at $\left.e e=100 \%\right)$ gives rise to a metastable type III solid solution according to Roozeboom classification), a stable racemic compound $\mathrm{RI}\left(\mathrm{mp}=161.3{ }^{\circ} \mathrm{C}\right.$ ) and a metastable type II solid solution ssRII ( $\mathrm{mp}=151.0^{\circ} \mathrm{C}$ at $e e=0 \%$ ). Structural determinations of $\mathrm{EI}, \mathrm{RI}$ and ssRII evidenced that the molecular flexibility of the propanediol substituent (carrying the only two $\mathrm{H}$-bond donor groups of the molecule) was at least partially responsible for this diversity of crystal forms.

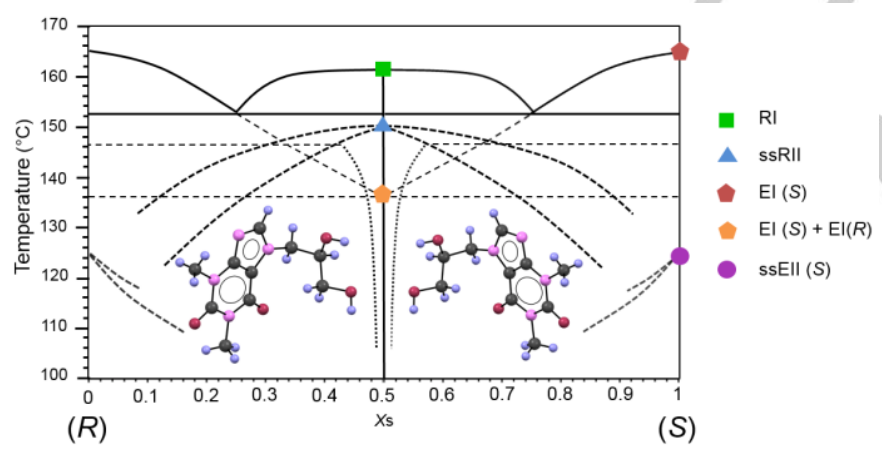

Figure 2. Schematic phase diagram between DPL enantiomers depicting stable (full lines) and metastable (dotted lines) equilibria.

As a result of this conformational variability, crystallization experiments of racemic DPL from highly supersaturated solutions using polar solvents (i.e., water, dimethylsulfoxide and dimethylformamide) systematically resulted in large metastable zone widths (MSZW), indicative of the poor nucleation rate of DPL in such solutions, ${ }^{[44]}$ which allowed the successful chiral resolution of racemic DPL by preferential crystallization. ${ }^{[43]}$ The system of $(R S)$-DPL enantiomers combines the three possible situations: stable racemic compound, metastable conglomerate and metastable solid solution. In the present contribution, this unique opportunity is exploited so as to compare the specific features of the solid solution ssRII with those of the racemic compound $\mathrm{Rl}$ in terms of chiral discrimination during crystallization. To this end, our experimental approach consisted in analysing sequentially the different stages of the crystallization process, i.e. pre-nucleation arrangement and nucleation, as well as crystal growth, by using a suitable combination of analytical techniques. Chiral discrimination mechanisms at the molecular sites scale are further rationalized with the help of modelling tools at the molecular mechanics level of theory.

\section{Results and Discussion}

As stated in previous papers, ${ }^{[43,50]}$ the stable racemic compound (RI) of Diprophylline (DPL) is produced by slow cooling crystallization from seeded solutions prepared with most organic solvents while the metastable racemic solid solution (ssRII) was reproducibly obtained either by crystallization at 90 $100^{\circ} \mathrm{C}$ from the supercooled melt (SCM) or by seeding a supersaturated 2-propanol (IPA) solution. Furthermore, it was observed that seeding a supersaturated (relative supersaturation $\beta=C / C_{s}>3$, with $C$ the actual concentration and $C_{s}$ the solubility at the seeding temperature) dimethylformamide (DMF) solution with the pure enantiomer $\mathrm{El}$ is suitable for preferential crystallization of this single enantiomer in quasi-racemic conditions. These two solvents (DMF and IPA) were therefore selected for further investigations of the crystallization behaviour of racemic DPL.

Table 1. Summary of the results obtained for the seeded and unseeded crystallizations of racemic DPL from IPA and DMF supersaturated solutions with $\beta=3$.

\begin{tabular}{lcccc}
\hline Nature of the seeds & RI & ssRII & EI & - \\
\hline Crystallization from IPA & ssRII & ssRII & ssRII & ssRII \\
Crystallization from DMF & RI & RI+ssRII & EI & RI $^{[a]}$
\end{tabular}

${ }^{[a]}$ long induction time observed (usually more than 3 hours)

A series of crystallization experiments in these two solvents was performed and XRPD analyses of the crystallized materials revealed that seeding a clear supersaturated solution in IPA prepared with racemic DPL $(\beta=3)$ reproducibly produces polymorph ssRII whatever the nature of the seeds inoculated (i.e., RI, ssRII or El). Moreover, unseeded IPA solutions spontaneously gave rise to ssRll within minutes once supersaturation (i.e., $\beta=3$ ) was established. By contrast, the polymorphic outcome from a similarly supersaturated DMF solution was shown to be seed-dependent (Table 1, XRPD are shown as Supporting Information) while unseeded DMF solutions exhibited long induction times (up to 3 hours) and spontaneously produced form RI. In terms of kinetics of spontaneous nucleation, it has recently been reported ${ }^{[44]}$ that the nucleation rate of ssRII from unseeded IPA solution at $\beta=3$ 
$\left(\mathrm{J}=668.0 \mathrm{~m}^{-3} \cdot \mathrm{s}^{-1}\right)$ is approximately 18 times higher than that of $\mathrm{RI}$ in unseeded DMF $\left(\mathrm{J}=35.7 \mathrm{~m}^{-3} \cdot \mathrm{s}^{-1}\right.$, at the same supersaturation).

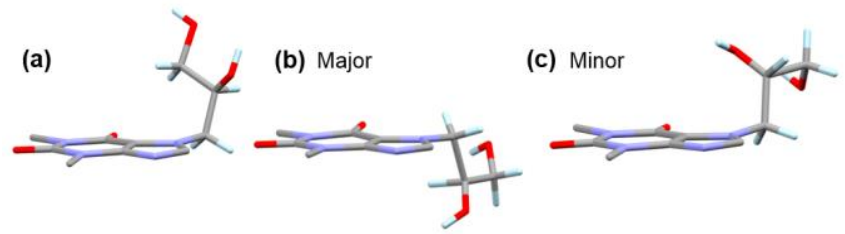

Figure 3. Molecular conformations of the $S$ enantiomer in the crystal structures of Form RI (a) and Form ssRII (b and c). In the latter case, the different conformations (major - $M$ conformation (b) and minor - $m$ conformation (c)) possible for a single enantiomer is shown. Hydrogen atoms have been removed for clarity.
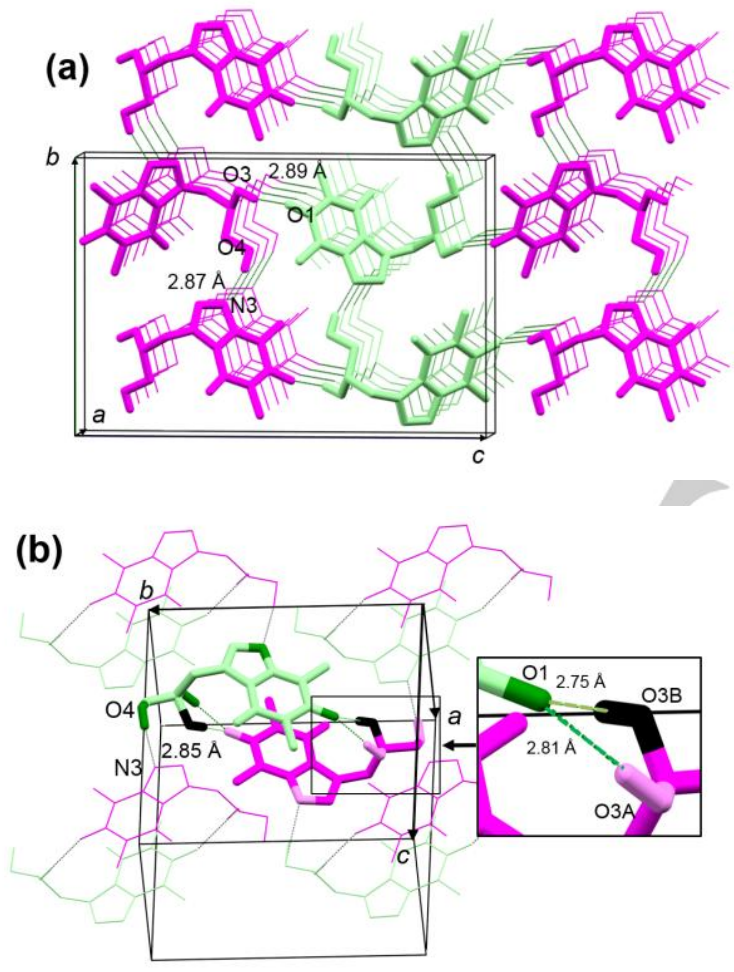

Figure 4. Representations of the packing and $\mathrm{H}$-bond pattern in $\mathrm{RI}$ (a) and in ssRII (b) (pink= $R$ enantiomer, green=S enantiomer). Non $\mathrm{H}$-bonding atoms have been removed for clarity, main $\mathrm{H}$-bond distances are given and the minor enantiomer in the packing of ssRII is shown with black atoms.

The main structural characteristics of $\mathrm{RI}$ and ssRII, thoroughly reported elsewhere, ${ }^{[43]}$ are presented in this paper for the sake of convenience. Figure 3 shows the different conformational features of the propanediol substituent of the DPL molecule (the $S$ enantiomer is arbitrarily chosen for comparative purpose) observed in the two racemic structures. In ssRII, both enantiomers can occupy any crystallographic site with an approximate occupancy factor of $80 / 20$ (a ca. 4:1 ratio of opposite enantiomers on each molecular site), a phenomenon referred to as enantiomeric disorder, ${ }^{[24]}$ which is in relation with the solid solution nature of this phase. The major $(\mathrm{M})$ and minor (m) conformations possible for a single enantiomer are shown in Figure $3 \mathrm{~b}$ and $\mathrm{c}$. Figure 4 shows the hydrogen-bond networks of $\mathrm{RI}$ and ssRII. In RI, the crystal structure results from the stacking along the $a$ axis of (100) heterochiral slices of low energy (Figure 4a). Concerning ssRII, the enantiomer with a minor occupancy is shown as black atoms in Figure 4b. It is worth noting that the DPL dimers in the ssRII crystal structure via O3$\mathrm{H} \cdots \mathrm{O} 1$ interactions can be either heterochiral (i.e., centrosymmetric $R_{\mathrm{M}}-S_{\mathrm{M}}$ associations) or homochiral (i.e., noncentrosymmetric $R_{M^{-}} R_{m}$ or $S_{M^{-}} S_{m}$ associations) as a result of enantiomeric disorder. In turn, each dimer is linked to four neighbouring dimers via $\mathrm{O} 4-\mathrm{H} \cdots \mathrm{N} 3$ intermolecular hydrogen bonds.

In order to understand the molecular mechanisms affecting the chiral discrimination phenomena, the DPL crystallization behaviour was further examined by an experimental study of the solvated state and of crystal growth while the enantiomeric selectivity was investigated using molecular modelling.

\subsection{The solvated state: Raman spectroscopy}

The distinct crystallization behaviours shown in Table 1 prompted us to investigate the solvated state of the supersaturated solutions by using Raman spectroscopy in an attempt to identify the origin of (i) the polymorphic selection from IPA and the related high nucleation rate of ssRII and (ii) the apparent difficult nucleation of solid phases from DMF with a very low nucleation rate of $\mathrm{RI}$.

Raman spectra of racemic DPL were collected at $20^{\circ} \mathrm{C}$ in the solid state (RI, ssRII and SCM) as well as in supersaturated DMF and IPA solutions (with comparable supersaturation ratio of $\beta \approx 3$ ). Based on the complete vibrational band assignment established for theophylline and caffeine, ${ }^{[51]}$ two regions of particular interest are shown in Figure 5. The 1550-1750 $\mathrm{cm}^{-1}$ region allows the monitoring of $(\mathrm{C}=\mathrm{O})$ and $(\mathrm{C}=\mathrm{N})$ stretching $(\mathrm{stg})$ bands, both involved as acceptors in intermolecular $\mathrm{H}$-bonding whereas the $1300-1500 \mathrm{~cm}^{-1}$ region depicts the contributions of $\mathrm{CH}_{2}$ deformations, sensitive to the molecular conformation of the propanediol substituent.

From the spectral features of the two crystalline forms, it appears that their distinct packings and conformational features result in different band positions and intensities for the $\mathrm{CH}_{2}$ region (Figure 5b), in agreement with the different crystal structures (Figure 4). Clear discrepancies are also observed for $(\mathrm{C}=\mathrm{O})$ and $(\mathrm{C}=\mathrm{N})$ stg bands since only single bands (at 1640 and $1690 \mathrm{~cm}^{-1}$ ) are obtained for ssRIl whereas the corresponding bands in $\mathrm{RI}$ exhibit marked shouldering (at 1655 and $1700 \mathrm{~cm}^{-1}$ ), indicative of a split for these vibration modes in the solid state (Figure 5a). The splitting of the $(\mathrm{C}=\mathrm{O})$ stg band is retrieved in the spectrum of the DMF solution with a larger magnitude, but is not visible either for the IPA solution or for the amorphous material (SCM), despite similar shifts toward higher frequencies of $(\mathrm{C}=\mathrm{O})$ and $(\mathrm{C}=\mathrm{N})$ stg bands. When considering the whole $1300-1750 \mathrm{~cm}^{-1}$ range, it is noteworthy that the Raman spectra obtained in IPA solution and for the SCM are similar (arrows in Figure 5b), mainly characterized by broad 
bands of relatively weak intensities. Interestingly, these two spectra also exhibit analogies with that of ssRII whereas major differences can be seen when comparing to the spectra obtained for RI and in a DMF solution.
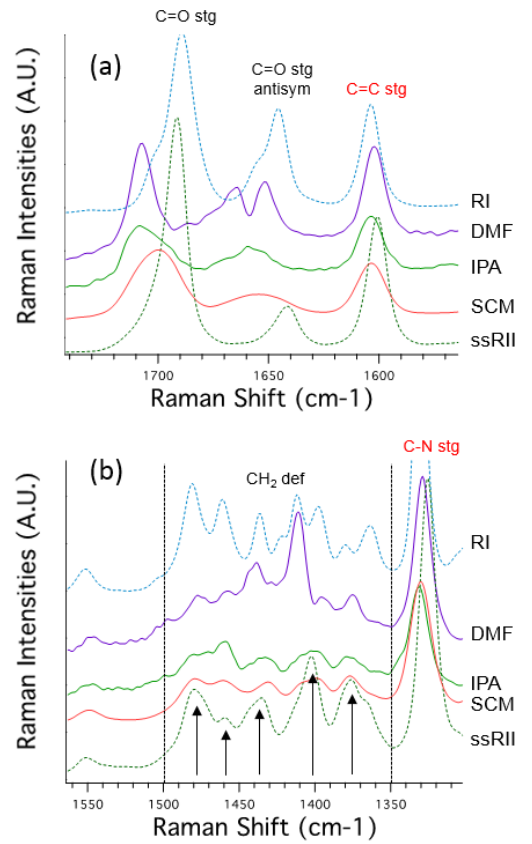

Figure 5. Raman spectra in the $1450-1750 \mathrm{~cm}^{-1}$ range (a), and in the 1300 $1550 \mathrm{~cm}^{-1}$ range (b) for solid forms RI, ssRII and SCM, and from supersaturated solutions in IPA (green) and DMF (purple). stg = stretching

From these spectroscopic analyses, although one can hardly establish the exact nature of intermolecular $\mathrm{H}$-bonding or possible self-associations pre-existing in the solvated or in the amorphous states, it can be deduced that molecular arrangements of DPL differ in the considered solvents.

In the case of DMF supersaturated solutions, the peak shifts and splitting observed for $\mathrm{C}=\mathrm{N}$ and $\mathrm{C}=\mathrm{O}$ stg vibrations cannot be due to interactions with the solvent and suggest that DPL self-association occurs, likely involving two populations of $\mathrm{H}$-bonded carbonyls, since both concerned sites (i.e., $\mathrm{C}=\mathrm{O}$ and $\mathrm{N})$ are $\mathrm{H}$-bond acceptors whereas DMF is not an $\mathrm{H}$-bond donor. Additionally ${ }^{1} \mathrm{H}$ NMR in DMF-d7 was used to measure chemical shifts of the DPL hydroxyl protons as a function of concentration (Supporting Information). The $\delta_{\mathrm{obs}}=\mathrm{f}\left(C_{\mathrm{DPL}}\right)$ curves showed the typical exponential decay expected for self-associated systems. ${ }^{[52]}$ However, it was impossible to fit these curves to different self-association models ${ }^{[53]}$ which may indicate the existence of at least two different populations of aggregates, or of a more complex degree of organization in this solvent. Such oligomeric associations and the conformational features of DPL in DMF depicted by the specific band pattern in the 1300-1750 $\mathrm{cm}^{-1}$ range (Figure $5 \mathrm{~b}$ ) might therefore be responsible for the rather difficult spontaneous nucleation in this solvent (Table 1). In the case of IPA (H-bond donor), the strongly broadened $\mathrm{C}=\mathrm{N}$ and $\mathrm{C}=\mathrm{O}$ stg peaks could be due to both the $\mathrm{H}$-bonding with the solvent and with other DPL molecules. However, these bands exhibit a similar profile in the spectrum of the SCM, thus suggesting that broadening is mainly due to DPL selfassociation in solution. Moreover, the similarities between the Raman spectra of IPA supersaturated solutions, in the molten state and for crystalline ssRII in the $1300-1750 \mathrm{~cm}^{-1}$ range (sensitive to the conformation of the propanediol substituent) indicate that DPL molecules adopt similar conformations in these different media. The existence of centrosymmetric dimeric associations in ssRII (Figure 4b) suggests that such preassociations might also exist in IPA solution and in the SCM, which appears consistent with the high crystallization tendency of ssRII from these media (Table 1). Unfortunately, due to fast proton exchange in 2-propanol-d8, it was not possible to study DPL self-association in this solvent by NMR spectroscopy.

Hence, it can be deduced from this spectroscopy study that (i) molecular associations (i.e. dimers) as well as conformations of DPL molecules in IPA supersaturated solutions and racemic SCM are probably similar to those adopted in SSRII, but that (ii) the conformation and presumed oligomeric associations of DPL molecules in DMF supersaturated solutions significantly differ from those adopted either in RI or in ssRII. These different solution chemistry features could be responsible for the different crystallization behaviours and kinetics of spontaneous nucleation reported above. As a complementary approach, the chiral discrimination mechanisms during crystal growth were investigated.

\subsection{Crystal growth study: morphology and composition of} ssRII single crystals

After investigation of the first two stages of DPL crystallization (pre-nucleation and nucleation) we turned our attention to the process of crystal growth in this system. In order to probe chiral selectivity at the molecular scale, a systematic crystal growth study from either racemic or enriched (ee=20\%) IPA solutions was carried out, aiming at evaluating the impact of the enantiomeric composition of the mother liquor on (i) the habit of the produced ssRII particles and (ii) the statistics of the enantiomeric composition of these crystals.

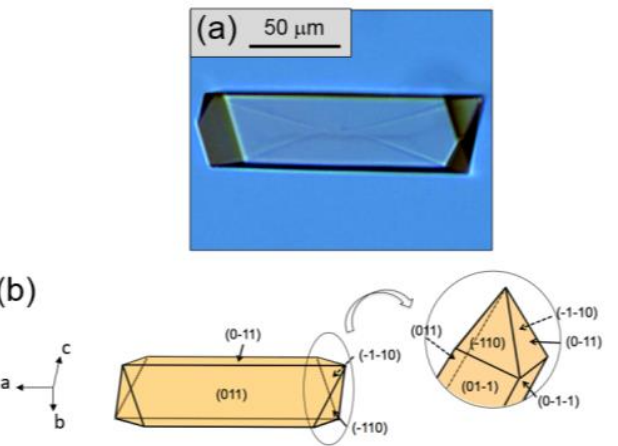

(b)

Figure 6. (a) Typical rod-shaped ssRII single crystal obtained from a racemic IPA solution. (b) Pictures of the simulated BFDH morphology of ssRII.

Starting from clear IPA solutions containing either ee=0\% (racemic composition) or $20 \%$, unstirred crystallization experiments were conducted by rapid cooling down to $20^{\circ} \mathrm{C}$ $(\beta=3)$ followed by seeding with ssRII. Figure 6 -a shows the 
typical rod morphology of a ssRIl single crystal grown from a quiescent racemic IPA solution. X-ray indexing of the crystal faces was confirmed by simulation of the equilibrium morphology by means of the BFDH method ${ }^{[47]}$ and indicated that the faces with largest morphological indexes are $\{011\}$ while the apex of the particle is made of $\{110\}$ faces (Figure $6-b$ ).
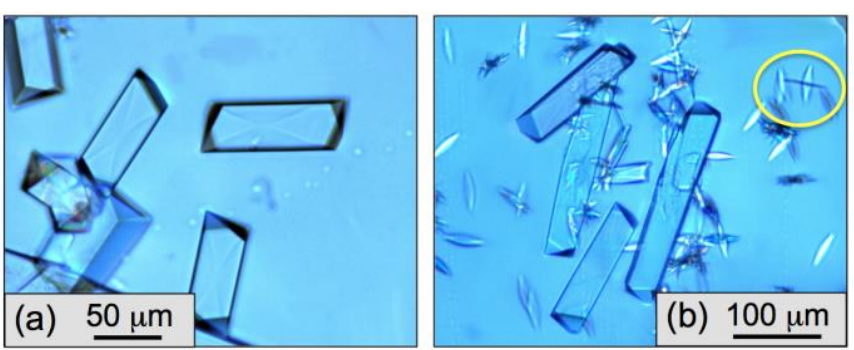

Figure 7. Optical microscopy pictures of ssRII particles grown from (a) racemic IPA solution and (b) from a $20 \%$ ee enriched IPA solution. In both cases, $T_{\text {growth }}=20^{\circ} \mathrm{C}$ and $\beta=3$. The yellow circle emphasizes the presence of $\mathrm{El}$ crystals.
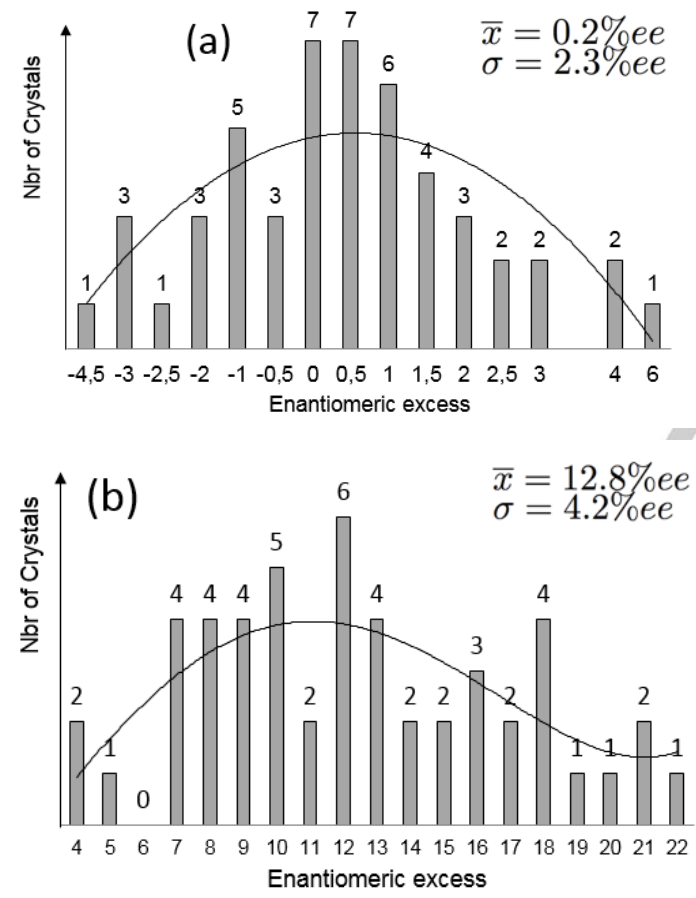

Figure 8. Results of the statistical studies for the enantiomeric composition of ssRII single crystals grown from IPA solutions prepared with (a) racemic DPL and (b) $20 \%$ ee enantio-enriched DPL.

For both experimental conditions (i.e., racemic or enantioenriched), kinetics of crystallization were found comparable since crystallizations were achieved within $2 \mathrm{~h}$ (note that $\mathrm{El}$ crystals, recognizable by their typical thin acicular morphology, were also obtained from enantio-enriched media as highlighted by the yellow circle in Figure 7-b). Interestingly, as depicted by the optical microscopy pictures of the two systems shown in Figure 7, it was reproducibly observed that ssRII crystals obtained in the enantio-enriched solutions are larger and significantly more elongated than those obtained from racemic composition, indicating either a faster growth of the $\{110\}$ faces in the enantio-enriched solution or a growth inhibition of the $\{011\}$ faces.

Statistically relevant set of data concerning the enantiomeric composition of the produced particles in both conditions were obtained by isolating and drying ca. 50 of the best shaped ssRII single particles crystallized in the two batches and determination by chiral HPLC of individual enantiomeric compositions. The results of these two statistical studies are shown in Figure $8 \mathrm{a}$ and $\mathrm{b}$ : a comparison between the two mean values (solid state $e e=0.2 \%$ and $12.8 \%$, the last value being significantly lower than the initial $20 \%$ ee in the supersaturated solution) highlights that the composition of the bulk crystallized materials depends on the composition of the saturated solution, but the enantiomeric composition of individual ssRII single crystals is governed by stochastic phenomena and cannot be predicted accurately. This is consistent with the solid solution nature of the ssRII phase (Figures 2 and 5) and confirms that the molecular sites of ssRIl can be occupied by both enantiomers although not fully randomly.

\subsection{Molecular modelling investigations}

\subsubsection{Adsorption of pre-associations at crystal surfaces}

Assuming that the growth units of this metastable phase consist of dimeric pre-associations as concluded from the spectroscopy study, basic molecular modelling calculations (molecular mechanics level of theory) were performed at the packing scale in order to account for the different observations made in this crystal growth study, i.e., (i) the elongation of ssRII particles in enantio-enriched IPA solutions (Figure 7) and (ii) the lack of chiral selection of the ssRII crystal structure (Figure 8), with the final aim to shed light on the molecular mechanisms of chiral discrimination in this system.

An assumption about the molecular mechanisms impacting the morphological features of ssRII single crystals as a function of the enantiomeric composition of the IPA solution (Figure 7) could be established through the calculation of the energy released during the incorporation of a single self-associated dimer in a vacant molecular site at either the $\{011\}$ or $\{110\}$ surfaces. Considering the 80/20 enantiomeric disorder observed in the ssRII crystal structure (Figure 4), we assumed the existence of two types of solvated aggregates in the mother liquor that result either from the $\mathrm{H}$-bond association of two DPL molecules of opposite chirality (i.e., heterochiral dimer $R_{\mathrm{M}^{-}} S_{\mathrm{M}}$, see Figure 3 ) or of the same chirality (i.e., homochiral dimer, $R_{\mathrm{M}^{-}}$ $R_{\mathrm{m}}$ or $S_{\mathrm{M}}-S_{\mathrm{m}}$, see Figure 3). A 50:50 distribution of such associations in the growth medium (i.e. $50 \%$ of heterochiral preassociations and $50 \%$ of homochiral ones) implies the existence of $50 \%$ of $R_{\mathrm{M}^{-}} S_{\mathrm{M}}, 25 \%$ of $R_{\mathrm{M}^{-}} R_{\mathrm{m}}$ and $25 \%$ of $S_{\mathrm{M}^{-}} S_{\mathrm{m}}$ preassociations at the solvated state. Therefore, in half of the cases, if a given molecular site is occupied by $R$ enantiomer with conformation $\mathrm{M}$, the site hosting the other constituent of the dimeric association will be occupied by $S$ enantiomer with conformation M. However, in the remaining halve of the cases accounting for the homochiral situations, the first molecular site is either occupied by $R_{\mathrm{M}}$ and forms a dimer with $R_{\mathrm{m}}$ on the opposite site or by $S_{\mathrm{m}}$ (which is isosteric to $R_{\mathrm{M}}$ ) in association with $S_{M}$. These different possible configurations give a 3:1 ratio 
of M:m (i.e. 75/25) conformations possible for a given molecular site in ssRII whereas the enantiomeric disorder observed upon structural refinement of ssRII was of $4: 1$ (i.e. $80 / 20$ ). Nevertheless, modelling calculations (first column of Table 2) indicated that heterochiral dimers are more stable than homochiral dimers (by ca. $4 \mathrm{kcal}^{\mathrm{mol}}{ }^{-1}$ ) and the actual $80 / 20(\mathrm{M} / \mathrm{m})$ ratio of enantiomeric disorder observed during crystal structure determination (Figure 4) could therefore result from a slightly larger proportion of heterochiral pre-associated species.

Both heterochiral and homochiral dimers were considered for the calculation of the energy released by the attachment of these building unit at the $\{011\}$ or $\{110\}$ crystal surfaces and the results of these calculations are summarized in Table 2. It can be concluded that both types of pre-associations release an energy of approximately $33.3 \mathrm{kcal} . \mathrm{mol}^{-1}$ when docked at the $\{110\}$ surface whereas the bounding of a homochiral dimer at the $\{011\}$ surface is unfavoured compared to the docking of a heterochiral dimer (ca. $7.7 \mathrm{kcal}^{\mathrm{mol}} \mathrm{l}^{-1}$ difference).

Table 2. Energies involved in docking of heterochiral and homochiral dimers in a vacant site at the $\{011\}$ and $\{110\}$ surface. All entries are in $\mathrm{kcal}^{\mathrm{mol}}{ }^{-1}$.

\begin{tabular}{lcc}
\hline & Surface $\{011\}$ & Surface $\{110\}$ \\
\hline Heterochiral dimer $(E=91.1)$ & $\Delta \mathrm{E}=22.2$ & $\Delta \mathrm{E}=33.3$ \\
Homochiral dimer $(\mathrm{E}=94.6)$ & $\Delta \mathrm{E}=14.5$ & $\Delta \mathrm{E}=33.3$ \\
\hline
\end{tabular}

Although many other parameters are overlooked in this procedure (such as the impact of solvation/desolvation energies), these results suggest that crystal growth at the $\{110\}$ faces is less stereoselective than that at the $\{011\}$ faces and might confirm that the growth mechanism via incorporation of the dimeric associations is relevant since faster $\{110\}$ growth rate is indeed observed in enantio-enriched medium (i.e., in the presence of a larger proportion of homochiral pre-associations in the solvated state). Concomitantly, our data also suggest that the growth rate of $\{011\}$ faces might be reduced by the presence of homochiral dimers in the enantio-enriched solution since their incorporation is less favourable than for heterochiral entities.

\subsubsection{Enantiomeric selectivity of the crystal lattice}

The steric and energetic consequences of the incorporation of a homochiral dimer in the structure of ssRII compared to the case of a heterochiral dimer were also considered to explain the lack of stereoselectivity of the ssRII molecular sites which can give rise to particles with composition as high as $6 \%$ ee in racemic solutions (Figure $8 a$ ). This was further investigated, using a similar modelling approach, by evaluating the effect of an enantiomeric substitution in a hypothetical structure, isomorphous to ssRII but built as a true racemic compound (i.e., made only of heterochiral associations). The procedure, described in the experimental section, consists in calculating the difference in lattice energy before and after enantiomeric substitution.

Figure 9-a shows a picture of the initial ssRII structure and associated periodic bond chains (PBC, yellow dashed lines) before enantiomeric substitution. The lattice energy has been computed and is reported in Table 3 . The chirality of the purple $S$ enantiomer in Figure 9-a has been switched to $R$ in Figure 9-b. After energy minimization of the system, one can see from Figure $9-b$ that the purple $R$ enantiomer adopts the conformation of the initial enantiomer. The torsion angles of the propanediol substituent of this $R$ enantiomer indicate a conformation similar to that of the minor conformer $m$ depicted in the crystallographic analysis of ssRII (Figure 3), and the permutation preserves the $\mathrm{O} 3 \mathrm{H} \cdots \mathrm{O} 1 \mathrm{H}$-bond (purple dashed line). As reported in Table 3, the energetic cost $\Delta U$ of this "chiral switch" in ssRII is as low as $0.7 \mathrm{kcal} / \mathrm{mol}$. This $\Delta U$ value was compared with those found when applying the same procedure in the RI and El crystal structures. The substitution performed in the structure of the stable racemic compound $\mathrm{RI}$ (Figure 9-c and d) afforded a $\Delta U$ value of $2.3 \mathrm{kcal} / \mathrm{mol}$ and the PBC was clearly disrupted as a result of the formation of a $\mathrm{O} 3 \mathrm{H} \cdots \mathrm{O} 4$ instead of a $\mathrm{O} 3 \mathrm{H} \cdots \mathrm{O} 1 \mathrm{H}$ bond with another neighbouring molecule. For the pure enantiomer $\mathrm{El}$, the chiral switch costs the system $2.4 \mathrm{kcal} / \mathrm{mol}$ and it can be seen from Figure 9-e and $f$ that the PBC is also disrupted: the permuted enantiomer establishes an intramolecular $\mathrm{O}_{3} \mathrm{H}^{\cdots} \mathrm{O} 2$ bond. Thus, the energy required for a chiral switch in ssRII is roughly three times smaller than in the case of the stable racemic compound RI and of the pure enantiomer El. This is consistent with both the solid solution nature of ssRII and the absence of a solid solution form isomorphous to $\mathrm{El}$ and $\mathrm{RI}$ (Figure 2). It also indicates that homochiral dimers can be incorporated in the lattice of ssRII without high energetic cost compared to a heterochiral one and underlines that both dimeric pre-associations satisfy Chion's criteria of isosterism and isomorphism ${ }^{[22]}$ for the formation of a solid solution.

\section{Conclusions}

The crystallization behaviour of racemic DPL from two different solvents was investigated using a methodological approach focusing on the different steps of the crystallization processes (pre-nucleation, nucleation, and crystal growth). The large metastable zone widths observed in dimethylformamide (DMF) supersaturated solutions contrasted interestingly with the fast and systematic crystallization of the metastable solid solution ssRII from supersaturated 2-propanol (IPA) solutions. The Raman analyses of these two supersaturated solutions highlighted the existence of self-associated species in IPA solutions that are actually retrieved as building units in the crystal structure of ssRII whereas organization of DPL in DMF does not resemble any of the known crystal structures. In agreement with nucleation kinetics, this observation provides another example for which pre-associations in the solution could indeed be building units for clusters according to classical nucleation theory (CNT) to form nuclei with the same structure as that of the bulk crystal phase. Furthermore, pre-nucleation aggregates in IPA also play a major role from the point of view of chiral discrimination in this system since the lack of chiral 

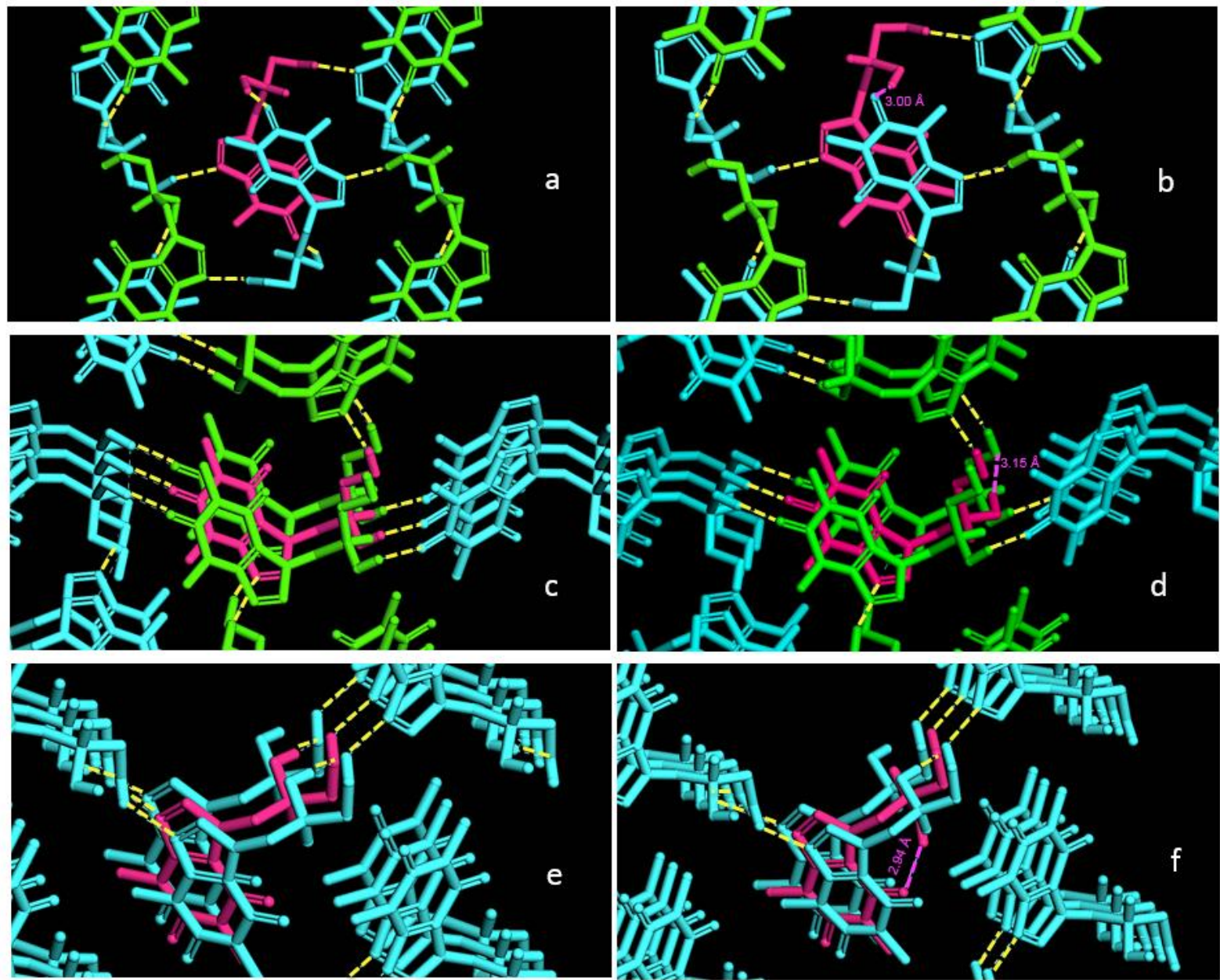

Figure 9. Representation of the reference structure (left) and the structure after permutation of the purple $(S)$ enantiomer into $(R)(R i g h t)$ for ssRII (a and b), RI (c and $\mathrm{d}$ ) and for $\mathrm{El}$ (e and f). In the coordination environment, the $(S)$ enantiomers are in green and $(R)$ enantiomers are in blue. Purple dashed lines correspond to the new set of $\mathrm{H}$-bonds generated after enantiomeric substitution.

Table 3. Energies involved in docking of heterochiral and homochiral dimers in a vacant site at the $\{011\}$ and $\{110\}$ surface. All entries are in $\mathrm{kcal}^{\mathrm{m}} \mathrm{mol}^{-1}$.

\begin{tabular}{|c|c|c|c|c|c|c|}
\hline & & $E$ & $E_{\text {host }}$ & $E_{\text {guest }}$ & $U_{\text {lattice }}$ & $\Delta U$ \\
\hline \multirow{2}{*}{$\mathrm{RI}$} & Reference & 2164.9 & 2160.1 & 56.2 & $\begin{array}{l}-51.4 \\
\end{array}$ & \multirow{2}{*}{+2.3} \\
\hline & Solid solution & 2170.0 & 2162.4 & 56.7 & -49.1 & \\
\hline \multirow{2}{*}{ ssRII } & Reference & 2022.6 & 2017.0 & 54.7 & -49.0 & \multirow{2}{*}{+0.7} \\
\hline & Solid solution & 2023.4 & 2016.8 & 54.8 & -48.4 & \\
\hline \multirow{2}{*}{$\mathrm{El}$} & Reference & 2279.0 & 2271.3 & 56.9 & -49.2 & \multirow{2}{*}{+2.4} \\
\hline & Solid solution & 2282.5 & 2272.3 & 57.0 & -46.9 & \\
\hline
\end{tabular}

selectivity of ssRII results from the random incorporation of solvated pre-associations (i.e., either homo or heterochiral dimeric associations) during crystal growth that are actually isosteric with reference to the crystal packing. This study therefore underlines the necessity to investigate solution chemistry in order to further understand the structural aspects of solid solutions between enantiomers. In this regard, the methodological approach developed in the present publication which focuses on the mechanisms of chiral selectivity during crystallization of a metastable solid solution down to its prehistory might be relevant for the rationalization of more specific phenomena, such as preferential enrichment. Thus, the methodological approach presented in this contribution might constitute a valuable input to rationalize versatile and complex behaviour during crystallization which could be considered to prevent solid solution formation upon processing the material. 


\section{Experimental Section}

Materials, Crystallization Methods, Solubility Measurements and Measurement of Nucleation Rates

All solvents used were of analytical grade and were purchased from Fisher Scientific (USA). Racemic DPL was purchased from SigmaAldrich (USA, HPLC purity > 99\%) and was recrystallized from pure ethanol prior to further treatments to afford polymorph $\mathrm{RI}$ as a white powder. Pure enantiomers $(S)$ - and $(R)$-DPL were synthesized as described elsewhere ${ }^{[43]}$ with ee HPLC $=99.9 \%$ and consist of form El after recrystallization from any commonly used solvent.

In order to obtain powdered samples of the metastable form ssRII, $30 \mathrm{~mL}$ of clear 2-propanol (IPA) solutions are prepared with $1.3 \mathrm{~g} \mathrm{DPL}$ (either racemic or with $20 \%$ ee) and homogenized by magnetic stirring at $70{ }^{\circ} \mathrm{C}$. Then, a rapid cooling down to $20^{\circ} \mathrm{C}$ is applied. Diffraction quality single crystals of ssRII can be prepared by keeping this supersaturated solution under quiescent conditions at room temperature.

All crystallization experiments were performed in $200 \mathrm{~mL}$ glass tubes, equipped with magnetic bottom stirrer. The temperature of the crystallizers was regulated $\left( \pm 1^{\circ} \mathrm{C}\right)$ using a Julabo F25 cryothermostat. The crystallized samples were Buchner filtered and dried in an oven at $50{ }^{\circ} \mathrm{C}$ for $12 \mathrm{~h}$ before further analyses.

Solubility measurements, performed in triplicate, were conducted by means of the gravimetric method after $12 \mathrm{~h}$ homogenisation of the saturated solutions at the considered temperature.

\section{Raman spectroscopy}

Raman spectra of supersaturated solutions or pure solids were measured with a Hololab series 5000 Raman spectrometer (Kaiser Optical System, Inc). Supersaturated solutions were prepared by dissolving a suitable mass of racemic DPL in $30 \mathrm{~mL}$ of either DMF or IPA in glass tubes placed in a thermostated jacket set at $75^{\circ} \mathrm{C}$. After cooling down to room temperature, the Raman signal were collected with a probe fixed on the side of the tubes. The signals of pure solvents (acquired at the same temperature) were manually subtracted from those of the supersaturated solutions. IC Raman software was used for data treatment and curve subtractions.

\section{$X$-ray diffraction and Crystal Indexation}

When required, DPL crystal forms were identified by routine XRPD using a D8 diffractometer (Bruker, Germany) equipped with a modified goniometer of reverse-geometry $(-\theta /-\theta)$ and a LynxEye® detector (Bruker Germany). ${ }^{[4]}$ Using $\mathrm{Cu} \mathrm{K} \mathrm{K}_{\alpha 1}(\lambda=1.54059 \AA)$ with a tube voltage and amperage set at $40 \mathrm{kV}$ and $40 \mathrm{~mA}$ respectively, diffraction patterns were collected with a step of $0.04^{\circ}(2 \theta)$ and a 4 s/step counting time from 3 to $30^{\circ}(2 \theta)$.

For crystal indexation of the experimental morphology, the selected DPL single crystal was stuck on a glass fiber and mounted on the full three circle goniometer of a Bruker SMART APEX diffractometer equipped with a CCD area detector (with $\mathrm{MoK}_{\alpha 1}=0.71071 \AA$ ). The SMART software was used to determine the cell parameters. ${ }^{[46]}$ Crystal faces of a representative ssRII single crystal were identified by determining the $(3 \times 3)$ matrix relating the coordinates of the crystallographic axes and the angles defining the orientation of the single crystal with reference to the X-ray beam. The results were compared with the morphology simulated using the Bravais-Friedel-Donnay-Harker (BFDH) method. ${ }^{[47]}$

\section{Chiral HPLC analysis (C-HPLC)}

Accurate enantiomeric excess measurements of DPL single crystals were performed by chiral high performance liquid chromatography (C-HPLC) using a CHIRALPAK®) IC column (DAICEL group, Chiral Technologies Europe), 250x4.6 mm. The mobile phase was a heptane:ethanol (7:3, v:v) mixture and the flow rate was $1 \mathrm{~mL} / \mathrm{min}$. The wavelength for UV detection was $273 \mathrm{~nm}$ and the temperature of the analysis was $25^{\circ} \mathrm{C}$. In these conditions, retention times are $16.6 \mathrm{~min}$ for $(R)$-DPL and $20.3 \mathrm{~min}$ for (S)-DPL. Analyses were carried out using a Finnigan Surveyor apparatus (Fisher Thermoscientific). The resolution was greater than 1.5 for all separations. The enantiomeric excess values were obtained by integrating and comparing peak areas of $(R)$-DPL and (S)-DPL, using the ChromQuest® software.

\section{Molecular Modelling procedures}

Molecular modelling calculations were performed within Material Studio v5.0. ${ }^{[48]}$ All atomic charges were computed by semi empirical calculations at the NDDO level of theory using the AM1 Hamiltonian in the VAMP module. For molecular mechanics procedures, the Dreiding forcefield $^{[49]}$ and the Smart algorithm were used with standard parameters for energy minimizations (i.e., 500 maximum iterations, medium convergence tolerance: $0.001 \mathrm{kcal} / \mathrm{mol}$ and $0.5 \mathrm{kcal} / \mathrm{mol} / \AA$ respectively set for energy and force). Prior to calculations, correction of the molecular geometry was performed by geometry optimization of the structural models obtained by SC-XRD, involving relaxation of the $\mathrm{C}-\mathrm{H}$ and $\mathrm{O}-\mathrm{H}$ bond distances, as well as the geometry of the theophylline fragment.

The energy released by the incorporation of a DPL dimeric preassociation at a crystal interface was calculated by first generating a supercell of ssRII that was then cleaved along the relevant directions (either $\{110\}$ or $\{011\}$ ). Then, a single dimeric unit was removed from the generated surface. Separately, structural models of dimeric associations (either homo or heterochiral) were extracted from the ssRII crystal structure. After energy minimization of the models, the energetic calculations consisted in comparing the energy of the selected surface and unbound dimer with that of the system in which the dimer is docked in the void previously created at the crystal surface. Thus, an estimate of the energy released during the integration of the dimers at the two types of crystal surface could be established.

Hypothetic solid solutions isomorphous to $\mathrm{El}, \mathrm{RI}$ and ssRII were modelled using the corresponding experimental crystal structures as reference situations and the viability of these hypothetical solid solutions was assessed by the following energetic calculations:

(i) El, RI and ssRII supercells of 64 DPL molecules were constructed and the symmetries were reduced to $P 1$. The energies of these 3 supercells $\left(E_{\mathrm{r}}\right)$ were subsequently computed.

(ii) The lattice energies, $U_{\text {r., }}$ of the 3 reference structures $\mathrm{EI}, \mathrm{RI}$ and ssRII were calculated. For this purpose, a fully coordinated molecule among the 64 molecules in the supercell was randomly removed. Then, the energy of the resulting lattice $\left(E_{\mathrm{l}}\right)$, and that of the isolated molecule $\left(E_{\mathrm{m}}\right)$, were calculated and compared to the energy of the initial lattice $\left(E_{\mathrm{r}}\right) . U_{\mathrm{r}}$ is then given by:

$$
U_{\mathrm{r}}=E_{\mathrm{r}}-\left(E_{\mathrm{l}}+E_{\mathrm{m}}\right)
$$

(iii) In the 3 supercells, one of the 64 molecules was manually substituted with the opposite enantiomer (the "guest") while preserving as far as possible the periodic bond chains of the structure and torsion geometries of the propanediol substituent. The geometries of the resulting hypothetic 
solid solutions were optimized (by minimizing the energy of the whole structure without any rigid body) and their respective energies $\left(E_{\mathrm{s}}\right)$ were computed.

(iv) Similarly to the above procedure, the lattice energies of the modelled solid solutions $\left(U_{s}\right)$ were estimated by calculating the energy of the isolated "guest" enantiomer $\left(E_{\mathrm{g}}\right)$ and that of the "host structure" $\left(E_{\mathrm{h}}\right)$. The lattice energy of solid solutions was then obtained by simple substraction:

$$
U_{\mathrm{s} .}=E_{\mathrm{s}}-\left(E_{\mathrm{h}}+E_{\mathrm{g}}\right)
$$

(v) The energetic cost for such enantiomeric substitution is related to the amount of additional energy generated by the guest enantiomer with its crystal environment, compared to the real structure. Therefore, based on structural considerations only, $\Delta U$ which is given by $\Delta U=U_{s}-U_{r}$ describes the energetic cost for the formation of a solid solution.

\section{Acknowledgements}

The authors would like to acknowledge networking support by the COST Action CM1402 "Crystallize". Samir Kulkarni is acknowledged for his help regarding experimental implementation of Raman spectroscopy and interpretation of Raman data. C. Brandel highly appreciated the hospitality that he received during his visit to the former crystallization group of J.H ter Horst at the Delft University of Technology.

Keywords: Chirality, Molecular Modeling, Crystal Growth, Enantiomeric Solid Solution, Pre-Nucleation Aggregates.

\section{References}

[1] J. W. Mullin, Crystallization, Butterworth Heinemann, 2001

[2] H.-H. Tung, E. L. Paul, M. Midler, J. A. McCauley, Crystallization of Organic Compounds: An Industrial Perspective, Hoboken (NJ), 2009.

[3] J. Jacques, A. Collet, S. H. Wilen, Enantiomers, Racemates and Resolutions, Krieger Publishing Company, Malabar, Florida, 1994

[4] G. Coquerel, Enantiomer 2000, 5, 481-498.

[5] R. M. Kellogg, M. Leeman in Comprehensive Chirality, Industrial Applications of Asymmetric Synthesis, Vol. 9 (Eds.: E. M. Carreira, H. Yamamoto), Elsevier, Amsterdam, 2012, pp 367-399.

[6] H. W. B. Roozeboom, Z. Phys. Chem. 1899, 28, 494-517.

[7] Y. Wang, A. M. Chen, Org. Proc. Res. Dev. 2008, 12, 282-290.

[8] Z. J. Li, D. J. W. Grant, J. Pharm. Sci. 1997, 86, 1073-1078.

[9] A. Burger, J. M. Rollinger, P. Brüggeller, J. Pharm. Sci. 1997, 86, 674679.

[10] M. Matell, Ark. Kemi 1952, 5, 17-37.

[11] H. Kaemmerer, H. Lorenz, S. N. Black, A. Seidel-Morgenstern, Cryst. Growth Des. 2009, 9, 1851-1862.

[12] H. Lorenz, J. Von Langermann, G. Sadiq, C. C. Seaton, R. J. Davey, A. Seidel-Morgenstern, Cryst. Growth Des. 2011, 1549-1556.

[13] G. G. Z. Zhang, S. Y. L. Paspal, R. Suryanarayanan, D. J. W. Grant, J. Pharm. Sci. 2003, 92, 1356-1366.

[14] A. A. Bredikhin, Z. A. Bredikhina, F. S. Akhatova, D. V. Zakharychev, E. V. Polyakova, Tetrahedron: Asymmetry 2009, 20, 2130-2136.

[15] S. Srisanga, J. H. ter Horst, Cryst. Growth Des. 2010, 10, 1808-1812.

[16] I. B. Rietveld, M. Barrio, B. Do, J.-L. Tamarit, R. Céolin, J. Phys. Chem. B 2012, 116, 5568-5574.

[17] L. Renou, T. Morelli, S. Coste, M.-N. Petit, B. Berton, J.-J. Malandain, G. Coquerel, Cryst. Growth Des. 2007, 7, 1599-1607.
[18] Z. J. Li, M. T. Zell, E. J. Munson, D. J. W. Grant, J. Pharm. Sci. 1999 88, 337-346.

[19] R. K. Hylton, G. J. Tizzard, T. L. Threlfall, A. L. Ellis, S. J. Coles, C. C Seaton, E. Schulze, H. Lorenz, A. Seidel-Morgenstern, M. Stein, S.L. Price, J. Am. Chem. Soc. 2015, 137, 11095-11104.

[20] C. P. Brock, W. B. Schweizer, J. D. Dunitz, J. Am. Chem. Soc. 1991 113, 9811-9820.

[21] A. A. Bredikhin, Z. A. Bredikhina, D. V. Zakharychev, Mendeleev Commun. 2012, 22, 171-180.

[22] B. Chion, J. Lajzéowicz, D. Bordeaux, A. Collet, J. Jacques, J. Phys. Chem. 1978, 2682-2688.

[23] R. A. Esteves de Castro, J. Canotilho, R. M. Barbosa, M. Ramos Silva, A. Matos Beja, J. A. Paixao, J. S. Redinha, Cryst. Growth Des. 2007, 7, 493-500.

[24] J. Huang, S. Chen, I. A. Guzei, L. Yu, J. Am. Chem. Soc. 2006, 128, 11985-11992.

[25] K. Marthi, S. Larsen, M. Ács, J. Bálint, E. Fogassy, Acta Crystallogr. 1994, B50, 762-771.

[26] H. L. de Diego, A. D. Bond, R. J. Dancer, 2011, 416, 408-416.

[27] A. A. Bredikhin, Z. A. Bredikhina, D. V. Zakharychev, A. T. Gubaidullin, R. R. Fayzullin, CrystEngComm 2012, 14, 648-655.

[28] C. Brandel, S. Petit, Y. Cartigny, G. Coquerel, Curr. Pharm. Des. 2016, in press.

[29] H. A. J. Oonk, J. Kroon, Acta Crystallogr. 1976, B32, 500-504.

[30] F. Baert, R. Fouret, H. A. J. Oonk, J. Kroon, Acta Crystallogr. 1978 B34, 222-226.

[31] S. Chen, H. Xi, R. F. Henry, I. Marsden, G. G. Z. Zhang CrystEngComm 2010, 12, 1485-1493.

[32] H. E. Gallis, P. J. Van Ekeren, J. C. Van Miltenburg, H. A. J. Oonk Thermochim. Acta 1999, 326, 83-90.

[33] J. Sañé, J. Ruis, T. Calvet, M. a. Cuevas-Diarte, Acta Crystallogr. 1997 B53, 702-707.

[34] L. Yu, S. M. Reutzel-Edens, C. A. Mitchell, E. Lilly, Org. Process Res. Dev. 2000, 4, 396-402.

[35] M. B. Hursthouse, L. S. Huth, T. L. Threlfall, 2009, 34, 1231-1240.

[36] J. Bernstein, R. Davey, J. Henck, Angew. Chem. Int. Ed.. 1999, 38 3440-3461.

[37] S. a Kulkarni, E. S. McGarrity, H. Meekes, J. H. ter Horst, Chem Commun. 2012, 48, 4983-4985.

[38] S. A. Kulkarni, S. S. Kadam, H. Meekes, A. I. Stankiewicz, J. H. ter Horst, Cryst. Growth Des. 2013, 13, 2435-2440.

[39] R. J. Davey, S. L. M. Schroeder, J. H. ter Horst, Angew. Chem. Int. Ed. 2013, 52, 2166-2179.

[40] R. A. Sullivan, R. J. Davey, G. Sadiq, G. Dent, K. R. Back, J. H. Ter Horst, D. Toroz, R. B. Hammond, Cryst. Growth Des. 2014, 14, 2689 2696

[41] I. Weissbuch, M. Lahav, L. Leiserowitz, Cryst. Growth Des. 2003, 3, 125-150.

[42] Gervais, C.; Beilles, S.; Cardinaël, P.; Petit, S.; Coquerel, G. J. Phys Chem. B 2002, 106, 646-652.

[43] C. Brandel, Y. Amharar, J. M. Rollinger, U. J. Griesser, Y. Cartigny, S Petit, G. Coquerel, Mol. Pharm. 2013, 10, 3850-3861.

[44] C. Brandel, J. H. ter Horst, Faraday Discuss. 2015, 179, 199-214.

[45] G. Coquerel, M. Sanselme, A. Lafontaine, Method and Measuring Scattering of $X$-Rays, Its Applications and Implementation Device, 2012, WO2012/136921 A1

[46] Bruker AXS Inc., Bruker SMART (5.059) and SAINT (6.01) 1997, Madison, Wisconin.

[47] J. D. H. Donnay, D. Harker, Am. Miner. 1937, 22, 446-467.

[48] Accelrys, Inc., Materials Studio, version 5.0 2009, San Diego, CA.

[49] S. L. Mayo, B. D. Olafson, W. A. Goddard, J. Phys. Chem. 1990, 94, 8897-8909.

[50] U. J. Griesser, M. E. Auer, A. Burger, Sci. Pharm. 1999, 67, 319-330.

[51] S. Gunasekaran, G. Sankari, S. Ponnusamy, Spectrochim. Acta. 2005, A61, 117-127. 
[52] A. Mattei, X. Mei, A.-F. Miller, T. Li, Cryst. Growth Des. 2013, 13, 33033307.

[53] R. A. Chiarella, A. L. Gillon, R. C. Burton, R. G. Davey, G. Sadiq, A Auffret, M. Cioffi, C. A. Hunter, Faraday Discuss. 2007, 136, 179-193. 
Entry for the Table of Contents (Please choose one layout)

Layout 1:

\section{FULL PAPER}

Text for Table of Contents

Layout 2:

\section{FULL PAPER}

Understanding chiral discrimination mechanisms during crystallisation of a chiral compounds: Based on experimental and modelling tools, a methodological approach is used to track the molecular mechanisms responsible for the occurrence of enantiomeric solid solutions down to crystal pre-history. This methodology might be helpful to prevent the formation of solid solutions and to develop robust crystallisation processes.
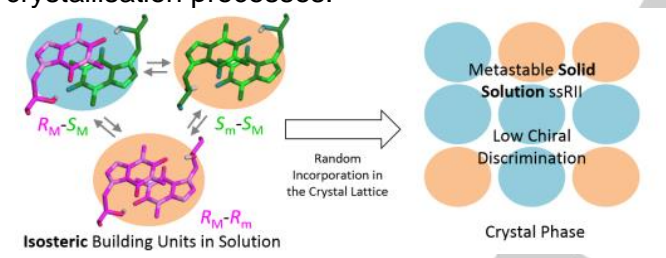

Author(s), Corresponding Author(s)*

Page No. - Page No.

Title

((Insert TOC Graphic here: $\max$. width: $5.5 \mathrm{~cm}$; max. height: $5.0 \mathrm{~cm}$ ))
C. Brandel, ${ }^{*}$ Y. Cartigny, G. Coquerel, J. H. ter Horst, S. Petit

Page No. - Page No.

Pre-Nucleation Self-Assembly and Chiral Discrimination Mechanisms during Solution Crystallization of Racemic Diprophylline 


\section{CHEMISTRY A European Journal}

\section{Supporting Information}

\section{Prenucleation Self-Assembly and Chiral Discrimination}

Mechanisms during Solution Crystallisation of Racemic Diprophylline

Clément Brandel, ${ }^{*[a]}$ Yohann Cartigny, ${ }_{1}^{[a]}$ Gérard Coquerel ${ }_{1}^{[a]}$ Joop H. ter Horst ${ }^{[b]}$ and Samuel Petit ${ }^{[a]}$

chem_201602707_sm_miscellaneous_information.pdf 


\section{CONTENTS :}

Figure S1. XRPD patterns of the crystals collected after crystallization of racemic DPL from IPA using different seeds.

Figure S2. XRPD patterns of the crystals collected after crystallization of racemic DPL from DMF using different seeds.

Figure S3. ${ }^{1} \mathrm{H}$ NMR chemical shifts of the DPL hydroxyl protons as a function of concentration in $d 7-D M F$.

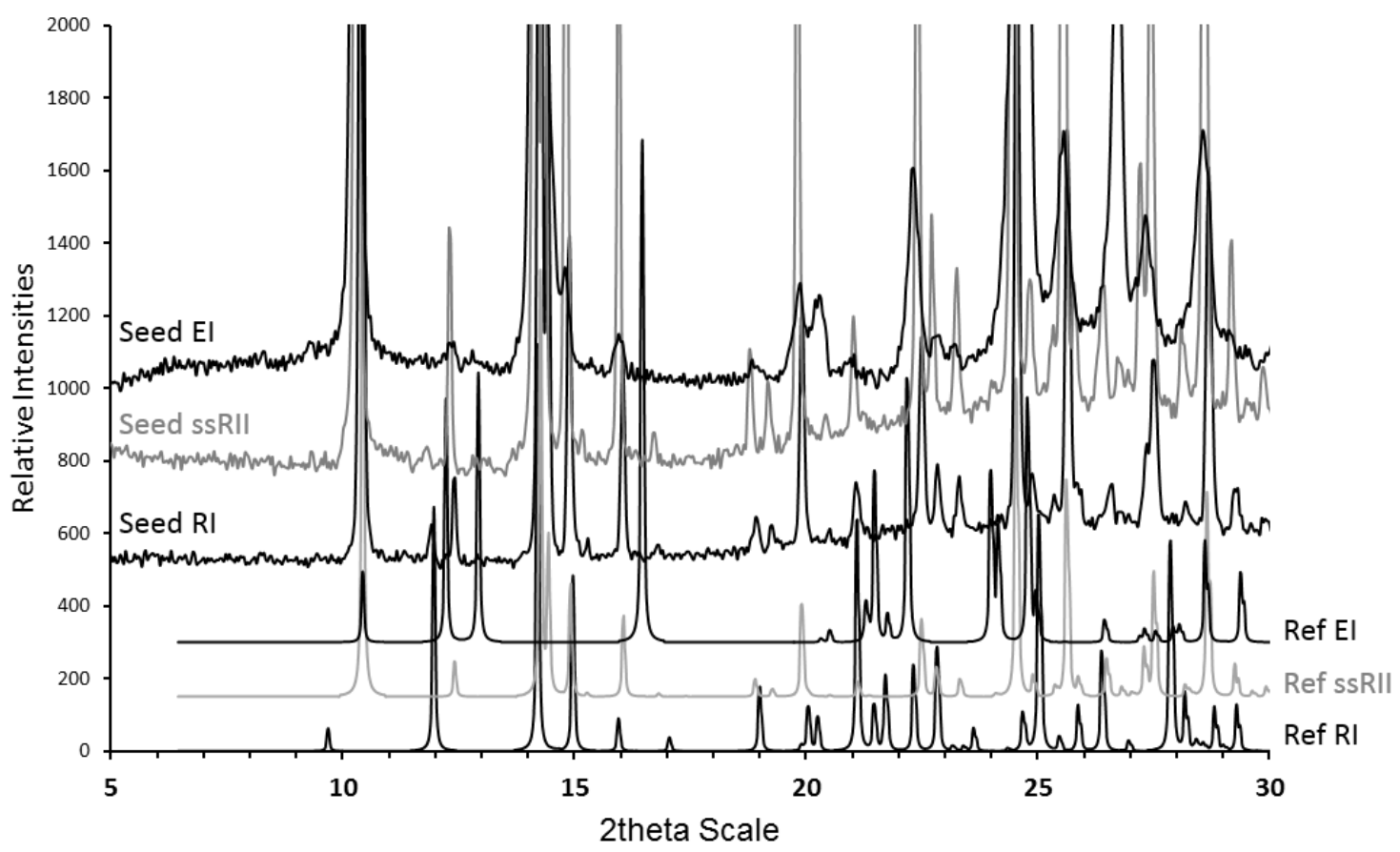

Figure S1. XRPD patterns of the crystals collected after crystallization of racemic DPL from IPA using different seeds. 


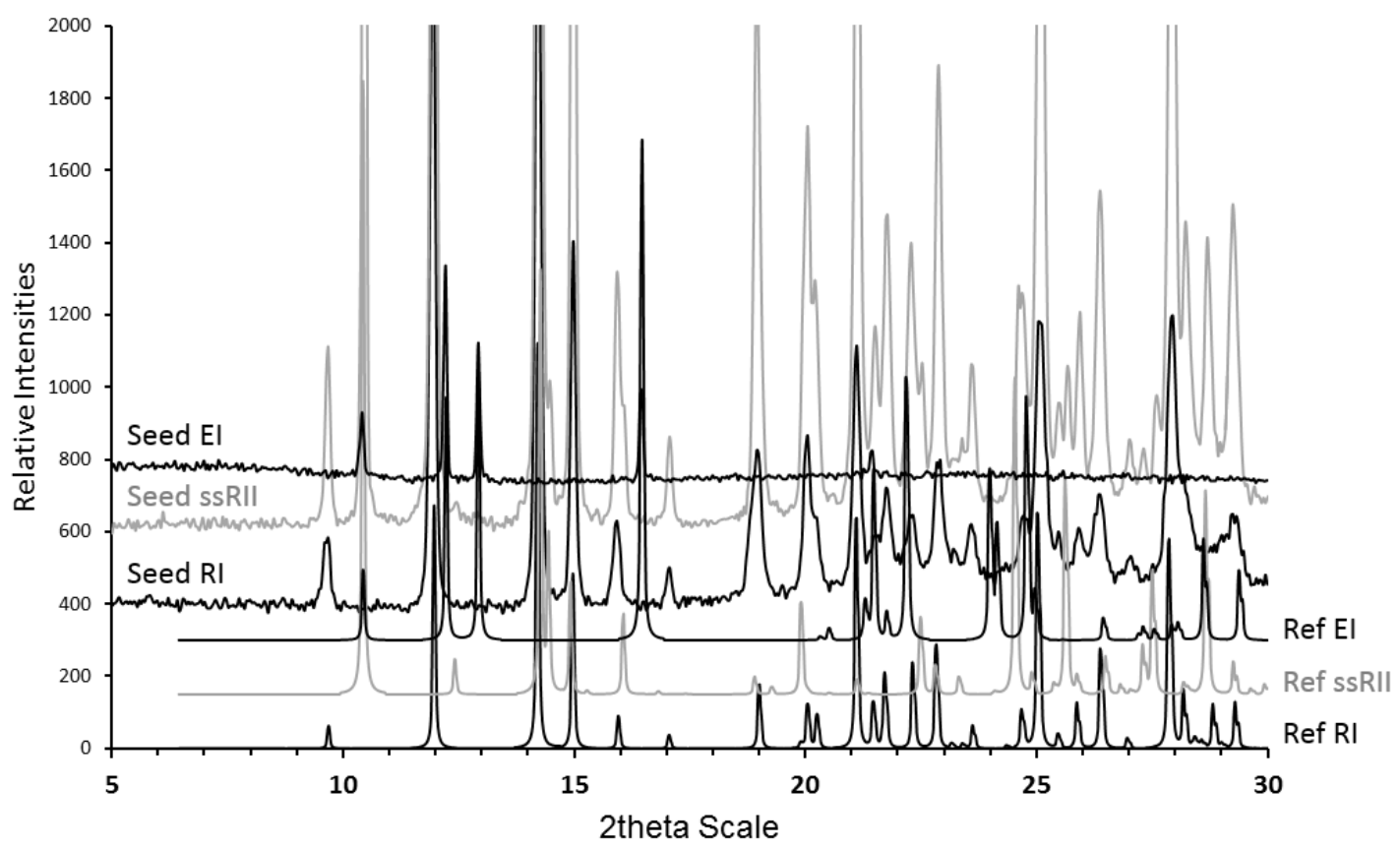

Figure S2. XRPD patterns of the crystals collected after crystallization of racemic DPL from DMF using different seeds.

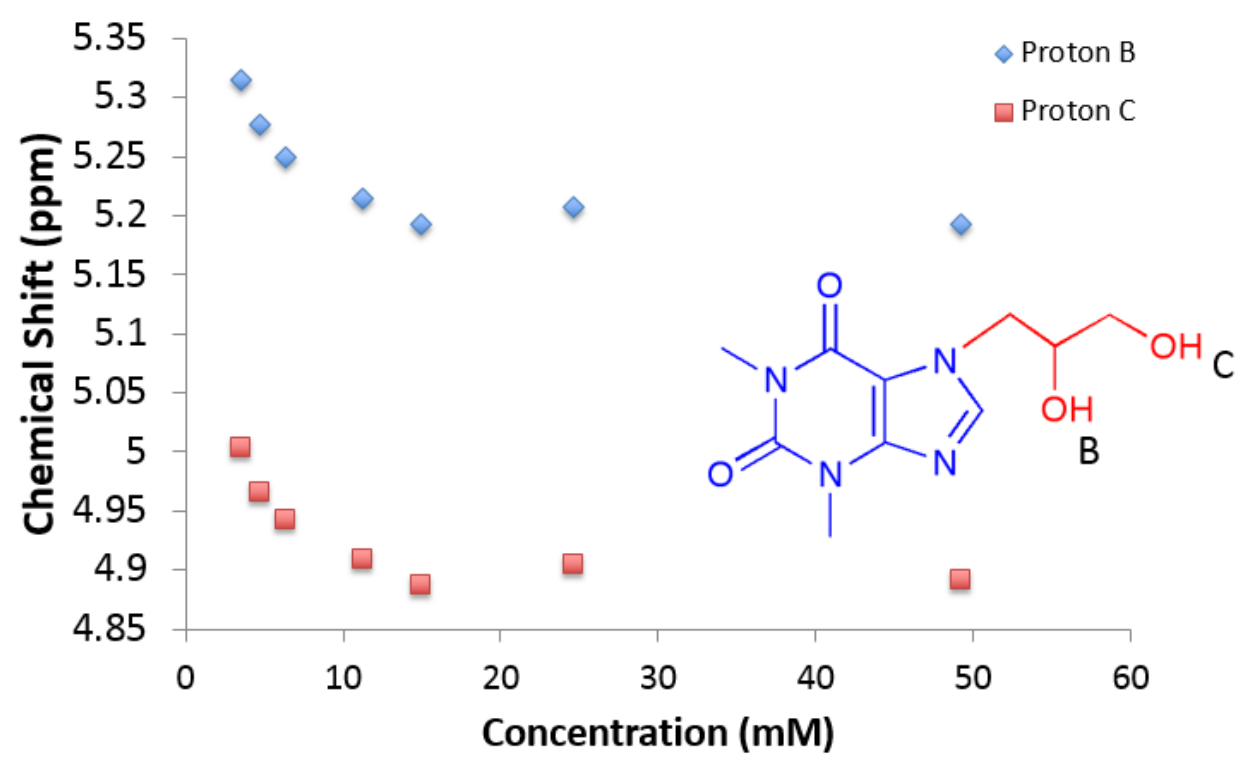

Figure S3. ${ }^{1} \mathrm{H}$ NMR chemical shifts of the DPL hydroxyl protons as a function of concentration in d7-DMF. 